\title{
AN EXTENSION OF A BEST APPROXIMATION THEOREM
}

\author{
A. CARBONE \\ Dipartimento di Matematica \\ Universitá della Calabria \\ 87036 Arcavacata di Rende (Cosenza), ITALY
}

(Received November 14, 1994 and in revised form February 10, 1995)

\begin{abstract}
In this paper an extension of a theorem of Prolla is given and several interesting corollaries are derived. Fixed point results are also given in the end.
\end{abstract}

KEY WORDS AND PHRASES. Almost affine map, coincidence point, approximatively compact set, fixed points.

1991 AMS SUBJECT CLASSIFICATION CODES. Primary 47H10, Secondary 54X10.

\section{INTRODUCTION.}

Recently several researchers have given extensions of the following well-known theorem of Ky Fan [4] on best approximation.

THEOREM 1. Let $C$ be a compact convex subset of a normed linear space $X$ and $f: C \rightarrow X$ a continuous function. Then there is an $x_{0} \in C$ such that

$$
\left\|x_{0}-f x_{0}\right\|=d\left(f x_{0}, C\right)=\inf \left\{\left\|f x_{0}-y\right\|: y \in C\right\} .
$$

There are several proofs of this theorem using the KKM-map principle, variational inequality and fixed point theory. The above theorem has interesting applications in fixed point theory and approximation theory.

Prolla [8] gave the following.

THEOREM 2. Let $C$ be a nonempty compact convex subset of a normed linear space $X$, and $g: C \rightarrow C$ continuous, almost-affine, onto map. If $f: C \rightarrow X$ is a continuous function then there is a $y_{0} \in C$ such that

$$
\left\|g y_{0}-f y_{0}\right\|=d\left(f y_{0}, C\right) .
$$

Note. In case $g=I$, an identity function, then Theorem 1 is obtained.

The purpose of this paper is to extend Theorem 2 and derive a few interesting corollaries.

We need the following.

Let $X$ be a Banach space and $C$ a nonempty subset of $X$. Let $x \in X$ and denote $d(x, C)=\inf \{\|x-y\|: y \in C\}$. In case $d(x, C)=\|x-y\|$ for $y \in C$, then $y$ is said to be an element of best approximation to $x$. The set of best approximation to $x$ is given by

$$
P(x)=\{y \in C:\|x-y\|=d(x, C)\} .
$$


The map $P: X \rightarrow 2^{C}$ is called the metric projection onto $C$. If $P x \neq \emptyset$ for all $x \in X$ then $C$ is called a proximinal set. In case $P(x)$ contains at most one element for each $x \in X$ then $C$ is called a Chebyshev set.

A subset $C$ of $X$ is called an approximatively compact if for every $x \in X$ and every sequence $\left\{y_{n}\right\}$ in $C$ with $\lim _{n \rightarrow \infty}\left\|x-y_{n}\right\|=d(x, C)$ there exists a subsequence $\left\{y_{n_{1}}\right\}$ converging to an element of $C$.

A compact set is always approximatively compact but converse is not true. For example, a closed convex set in a Hilbert space is approximatively compact but not compact.

For an approximately compact set $C$ the following holds.

i) $P x \neq \emptyset$ for each $x \in X$;

ii) $C$ is closed;

iii) $P x$ is compact;

iv) if $C$ is convex then $P x$ is convex;

v) the metric projection $P: X \rightarrow 2^{C}$ is upper semicontinuous (see [9] or [11]);

vi) $P(A)=\cup\{P(x): x \in A\}$ is compact for any compact subset $A$ in $X$.

Let $X$ and $Y$ be normed linear spaces and $2^{Y}$ denote the set of all nonempty subsets of $Y$. A multivalued mapping $F: X \rightarrow 2^{Y}$ is upper semicontinuous (usc) if $F^{-1}(A)=\{x \in X: F x \cap A \neq \emptyset\}$ is closed in $X$ for each closed set $A$ in $Y$.

A multivalued map $F$ is said to be compact if $F(X)$ is contained in a compact subset of $Y$. F is said to be acyclic if $F x$ is nonempty, compact and acyclic subset of $Y$ for each $x \in X$.

A multivalued map $F: X \rightarrow X(X$ is a metric space $)$ is said to be admissible if there are maps

$$
F_{i}: X_{i} \rightarrow X_{i+1} \quad i=0,1,2, \ldots, n \quad X_{0}=X_{n+1}=X
$$

such that:

i) $F=F_{n} F_{n-1} \ldots F_{0}$;

ii) $F_{i}$ is acyclic and usc for each $i$;

iii) $X_{i}$ are metric spaces for each $i=1,2, \ldots, n$ (see [7]).

The following theorem will be used [7] in our work.

THEOREM 3. Let $C$ be a convex subset of a Banach space $X$ and $F: C \rightarrow C$ an admissible compact map. Then $F$ has a fixed point.

Let $C$ be a convex subset of $X$. A map $g: C \rightarrow X$ is almost-affine if it satisfies

$$
\left\|g\left(\lambda x_{1}+(1-\lambda) x_{2}\right)-y\right\| \leq \lambda\left\|g x_{1}-y\right\|+(1-\lambda)\left\|g x_{2}-y\right\|
$$

for all $x_{1}, x_{2} \in C, y \in X$ and $0<\lambda<1$.

$g$ is an affine map if

$$
g\left(\lambda x_{1}+(1-\lambda) x_{2}\right)=\lambda g x_{1}+(1-\lambda) g x_{2}, \quad \lambda \in(0,1) .
$$

If $g: C \rightarrow C$ is a single-valued function then $g$ is said to be proper if $g^{-1}(A)$ is compact for $A$ compact.

The following is the main result.

THEOREM 4. Let $C$ be a nonempty convex subset of a normed linear space $X$ and $P: X \rightarrow 2^{C}$ the metric projection satisfying

i) $P(x)=\{y \in C:\|x-y\| \leq\|x-z\|$ for all $z \in C\} \neq \emptyset$ for each $x \in X$ and

ii) $P$ sends compact subsets of $X$ onto compact subsets of $C$.

Let $g: C \rightarrow C$ be a continuous, onto, proper and $g^{-1}(z)$ an acyclic subset of $C$ for every $z \in C$.

Then for every continuous map $f: C \rightarrow X$ with $f(C)$ relatively compact there exists a $y_{0} \in C$ such that 


$$
\left\|g y_{0}-f y_{0}\right\|=d\left(f y_{0}, C\right) .
$$

Note. 1. In case $C$ is an approximatively compact set then conditions i) and ii) are satisfied by $P$.

2. In case $C$ is a compact convex set then the condition that $f(C)$ is relatively compact is not required since the continuous image of a compact set is compact. The condition that $g$ is proper is also not needed, since $g$ is continuous so for any compact set $D$ in $C, g^{-1}(D)$ is a closed subset of a compact set $C$ and hence is compact.

The proof is on the same lines as in [3].

PROOF. Let $P: X \rightarrow 2^{C}$ be the metric projection. Define a multivalued map $F: C \rightarrow C$ by

$$
F x=\{y \in C: g y \in P(f(x))\} .
$$

Then $F x \neq \emptyset$ for each $x \in C$ since $g$ is an onto map. $F(x)$ is closed and acyclic (see [3]). We show that $F$ is upper semicontinuous. This will be done as in [10].

Let $B$ be a closed subset of $C$ and $z$ a limit point of $F^{-1}(B)$. We choose $\left\{z_{n}\right\} \subseteq F^{-1}(B) \subseteq C$ such that $z_{n} \rightarrow z$. We show that $z \in F^{-1}(B)$. Since for each $n, F\left(z_{n}\right) \cap B \neq \emptyset$ we have $\left\{y_{n}\right\} \subseteq C$ with $y_{n} \subseteq F\left(z_{n}\right) \cap B$.

Then for each $n$,

$$
\left\|g y_{n}-f z_{n}\right\|=d\left(f z_{n}, C\right) .
$$

Let $A=\overline{f(C)}$ and $D=P(A)$. By ii) $D$ is a compact subset of $C$. Since $g$ is a proper map $g^{-1}(D)$ is a compact subset of $C$.

Now, for each $n$,

$$
y_{n} \in F\left(z_{n}\right)=g^{-1} P f\left(z_{n}\right) \subseteq g^{-1} P f(C) \subseteq g^{-1}(D) .
$$

Consequently, there exists $y \in C$ and a subsequence $\left\{y_{n_{2}}\right\}$ of $\left\{y_{n}\right\}$ such that $y_{n_{4}} \rightarrow y$. By $(*)$ we get that

$$
\|g(y)-f(z)\|=d(f z, C) .
$$

This gives $y \in F(z) \cap B$, that is, $z \in F^{-1}(B)$ and $F$ is an upper semicontinuous multifunction.

Now, the map $F=g^{-1} \circ P \circ f: C \rightarrow C$ is an admissible map. Since $f(C)$ is relatively compact therefore $F(C)=g^{-1}(P(f(C)))$ is also relatively compact because the image of a compact set under upper semicontinuous map with compact values is compact.

Then $F$ satisfies conditions of Theorem 3 and has a fixed point, say $y_{0} \in F\left(y_{0}\right)$. This implies that

$$
\left\|g y_{0}-f y_{0}\right\|=d\left(f y_{0}, C\right) \text {. }
$$

An almost affine map satisfies the condition that $g^{-1}(z)$ is an acyclic set for each $z \in C$. As a consequence we have the following [10].

COROLLARY 1. Let $C$ be a nonempty convex subset of a normed linear space $X$ and $P: X \rightarrow 2^{C}$ the metric projection satisfying i) and ii) of Theorem 4. Let $g: C \rightarrow C$ be a continuous, onto, almost affine and proper map. Then for each continuous map $f: C \rightarrow X$ with $f(C)$ relatively compact there exists a $y_{0} \in C$ such that

$$
\left\|g y_{0}-f y_{0}\right\|=d\left(f y_{0}, C\right) .
$$

In case $C$ is an approximatively compact set then conditions i) and ii) are satisfied and we have the following [3].

COROLLARY 2. Let $C$ be an approximatively compact convex set of a normed linear space $X$ and $g: C \rightarrow C$ continuous, onto, proper and $g^{-1}(z)$ is an acyclic subset of $C$ for every $z \in C$. Then for each continuous function $f: C \rightarrow X$ with $f(C)$ relatively compact there exists an $x_{0} \in C$ such that 


$$
\left\|g x_{0}-f x_{0}\right\|=d\left(f x_{0}, C\right) .
$$

In case $C$ is an approximatively compact and $g=I$, an identity function, then we get a well-known result of Reich [9].

Recently in [2] almost quasi convex $g$ was considered.

The function $g: C \rightarrow X$ is said to be almost quasi convex if

$$
\left\|g\left(\lambda x_{1}+(1-\lambda) x_{2}\right)-y\right\| \leq \max \left(\left\|g x_{1}-y\right\|,\left\|g x_{2}-y\right\|\right)
$$

for $x_{1}, x_{2} \in C, y \in X$ and $0<\lambda<1$.

As in [3] it is easy to see that almost quasi convex implies that $g^{-1}(z)$ is an acyclic set. Therefore a recent result due to Park, Singh and Watson [6] given below, follows as a corollary.

COROLLARY 3. Let $C$ be a nonempty convex subset of a normed linear space $X$ and $P: X \rightarrow 2^{C}$ the metric projection satisfying

i) $P x \neq \emptyset$ for each $x \in X$, and

ii) $P$ sends compact subset of $X$ onto compact subsets of $C$.

Let $g: C \rightarrow C$ be a continuous, almost quasi convex, onto and proper map.

Then for each continuous map $f: C \rightarrow X$ with $\overline{f(C)}$ compact there is a $y_{0} \in C$ such that

$$
\left\|g y_{0}-f y_{0}\right\|=d\left(f y_{0}, C\right) \text {. }
$$

Note. They [6] concluded that either

i) $f$ and $g$ have a coincidence point $w \in C$, i.e., $f w=g w$, or

ii) there is a $w \in C$ such that $g w \in \partial C$ and

$$
0<\|g w-f w\| \leq\|z-f w\| \quad \text { for all } \quad z \in I_{C}(g w) .
$$

Recall that if $f: C \rightarrow X$ is a map, then the inward set $I_{C}(x)$, of $C$ at $x$ is defined by

$$
I_{C}(x)=\{y: y \in X \text {, there exist } u \in C \text { and } r>0 \text { such that } y=x+r(u-x)\} \text {. }
$$

The closure is denoted by $\overline{I_{C}(x)}$. The function $f$ is said to be inward map if $f(x) \in I_{C}(x)$ for every $x \in C$ and weakly inward if $f(x) \in \overline{I_{C}(x)}$.

In case $C$ is a compact convex subset of a normed linear space $X$ we get the following.

COROLLARY 4. Let $C$ be a compact convex subset of a normed linear space $X$ and $g: C \rightarrow C$ continuous, onto, $g^{-1}(z)$ is an acylic set for each $z \in C$. Then for each continuous function $f: C \rightarrow X$ there is a $y_{0} \in C$ such that

$$
\left\|g y_{0}-f y_{0}\right\|=d\left(f y_{0}, C\right) .
$$

If $g=I$, an identity function, in Corollary 4, then we get a well-known result of Ky Fan [4].

If in Corollary $4, g$ is almost-affine, continuous, onto then we get a theorem of Prolla [8].

If $f y_{0} \in C$ for all $y_{0}$ then Corollary 4 yields a coincidence theorem, that is, there is a $y_{0} \in C$ such that

$$
g y_{0}=f y_{0} .
$$

In case $g$ is an identity function in Corollary 4 and $f$ has an additional condition then we get a fixed point theorem given below.

COROLLARY 5. If all the hypotheses of Corollary 4 are satisfied with $g=I$, and in addition: for any $x \in \partial C$ with $x \neq f x$ there exists a number $\lambda$ (real or complex depending on whether the vector space is real or complex) such that $|\lambda|<1$ and 


$$
y=\lambda x+(1-\lambda) f x \in C,
$$

then $f$ has a fixed point.

PROOF. By Corollary 4 we have that

$$
\left\|y_{0}-f y_{0}\right\|=d\left(f y_{0}, C\right) .
$$

Take $y_{0} \in \partial C$ and assume that $y_{0} \neq f y_{0}$. Then

$$
\begin{aligned}
0<\left\|y_{0}-f y_{0}\right\| \leq\left\|y-f y_{0}\right\| & =\left\|\lambda y_{0}+(1-\lambda) f y_{0}-f y_{0}\right\| \\
& =\left\|\lambda\left(y_{0}-f y_{0}\right)\right\| \\
& =|\lambda|\left\|y_{0}-f y_{0}\right\|<\left\|y_{0}-f y_{0}\right\|
\end{aligned}
$$

a contradiction. Hence $y_{0}=f y_{0}$.

In case $g=I$, an identity function, then we could derive a very well-known result due to Browder [1] as a corollary.

COROLLARY 6. Let $X$ be a Banach space, $C$ a compact, convex subset of $X$ and $f: C \rightarrow X$ a continuous function. Suppose that for each $x \in C$ with $x \neq f x$ there exists a $y \in I_{C}(x)$ such that

$$
\|y-f x\|<\|x-f x\| \text {. }
$$

Then $f$ has a fixed point.

PROOF. By Corollary 4 (with $g=I$ ), we have

$$
\|x-f x\|=d(f x, C)
$$

i.e. $\|x-f x\| \leq\|f x-z\|$ for all $z \in C$.

This inequality remains valid (see [5]) for all $y \in I_{C}(x)$, i.e.

$$
\|x-f x\| \leq\|f x-y\| \quad \text { for all } \quad y \in I_{C}(x) .
$$

By hypothesis for each $x \in C$ with $x \neq f x$ we have $y \in I_{C}(x)$ such that

$$
\|y-f x\|<\|x-f x\|
$$

a contradiction to (**). Hence $f$ has a fixed point.

Further work in this direction due to Park [5] gives several applications.

\section{REFERENCES}

[1] BROWDER, F.E., On a sharpened form of the Schauder fixed point theorem, Proc. Natl. Acad. Sci. USA 74 (1977), 4749-4751.

[2] CARBONE, A., A note on a theorem of Prolla, Indian J. Pure Appl. Math. 23 (1991), 257-260.

[3] CARBONE, A. and CONTI, G., Multivalued maps and existence of best approximants, J. Approx. Theory 64 (1991), 203-208.

[4] FAN, K., Extensions of two fixed point theorems of F. E. Browder, Math. Z. 112 (1969), 234-240.

[5] PARK, S., On generalization of Ky Fan's theorem on best approximations, Numer. Funct. Anal. Optimiz. 9 (1987), 619-628.

[6] PARK, S., SINGH, S.P. and WATSON, B., Remarks on best approximations and fixed points, $J$. Pure Appl. Math. 25 (5) (1994), 459-462.

[7] POWERS, M.J., Leftschetz fixed point theorems for a new class of multivalued maps, Pacif. $J$. Math. 42 (1972), 211-220.

[8] PROLLA, J.B., Fixed point theorems for set-valued mappings and existence of best approximants, Numer. Funct. Anal. Optimiz. 5 (1982-83), 449-455. 
[9] REICH, S., Approximate selections, best approximations, fixed points, and invariant sets, J. Math. Anal. Appl. 62 (1978), 104-113

[10] SEHGAL, V.M. and SINGH, S.P., A theorem on best approximations, Numer. Funct. Anal. Optımiz. 10 (1989), 181-184

[11] SINGER, I., Best Approximations in Normed Linear Spaces by Elements of Linear Subspaces, Springer-Verlag (1970), 368 


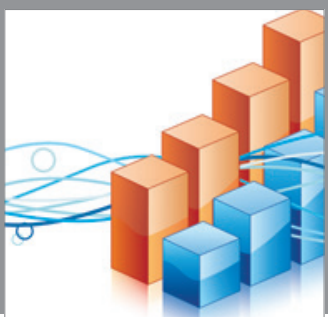

Advances in

Operations Research

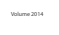

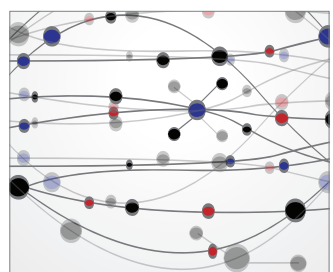

\section{The Scientific} World Journal
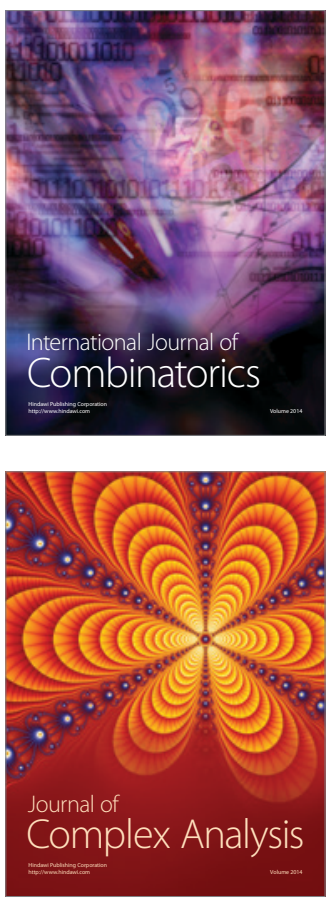

International Journal of

Mathematics and

Mathematical

Sciences
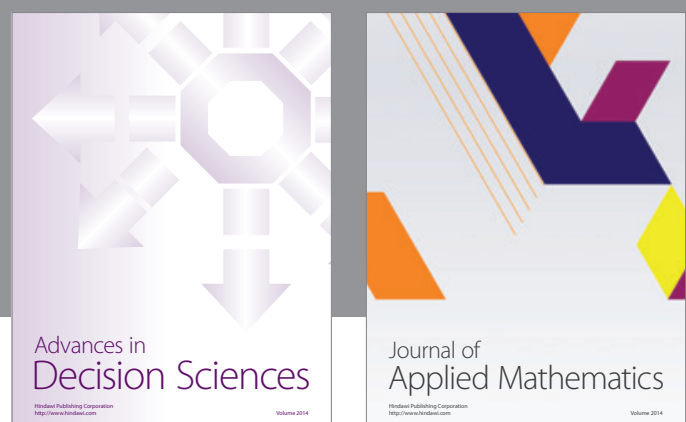

Journal of

Applied Mathematics
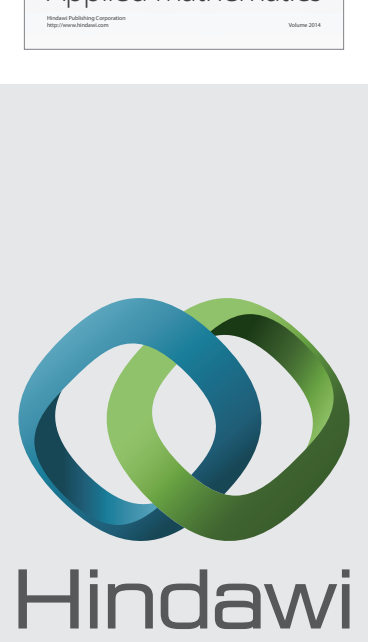

Submit your manuscripts at http://www.hindawi.com
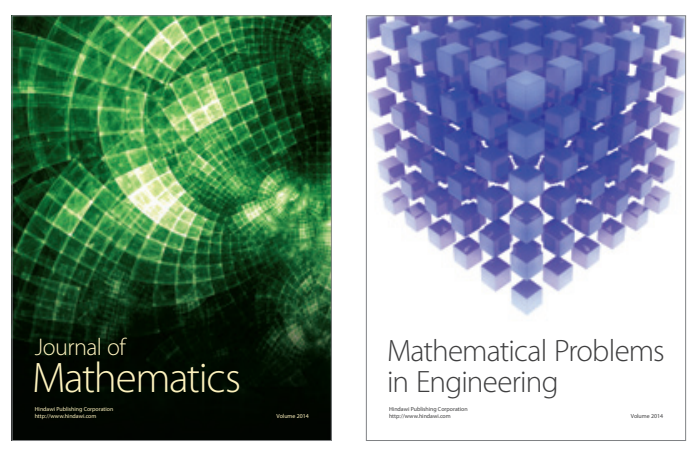

Mathematical Problems in Engineering
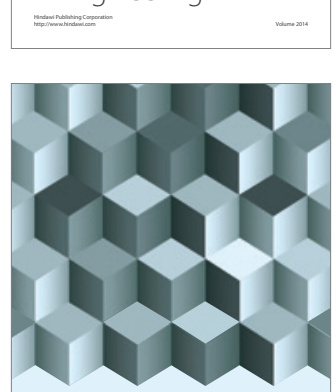

Journal of

Function Spaces
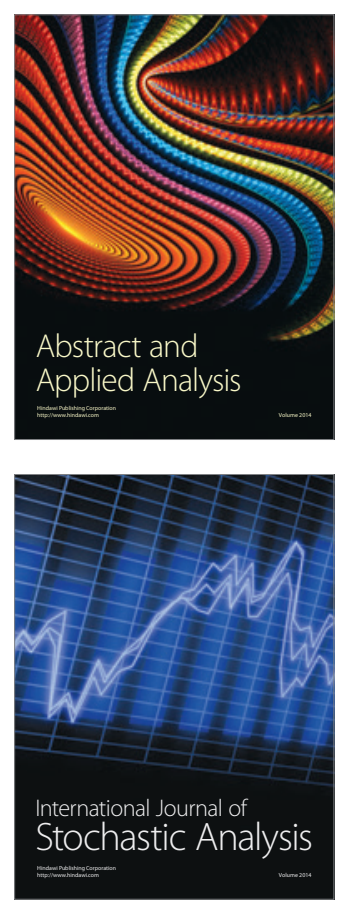

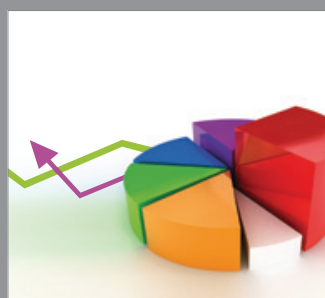

ournal of

Probability and Statistics

Promensencen
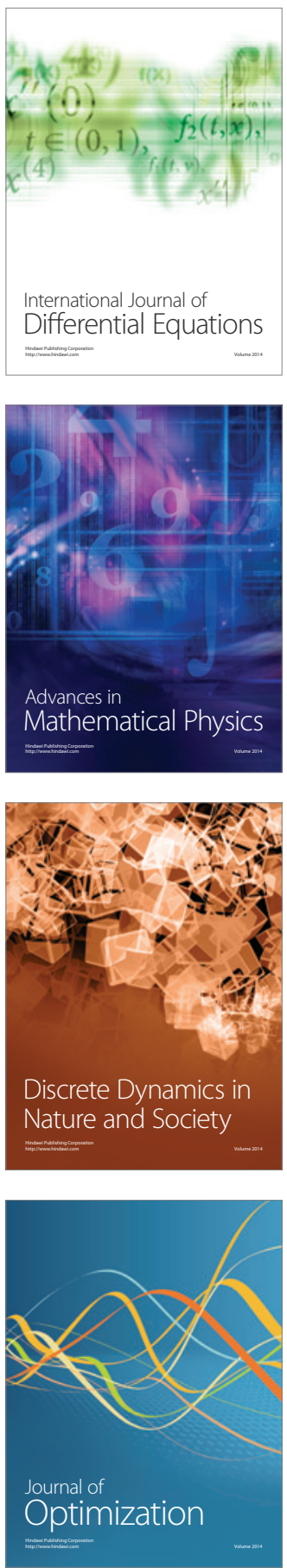